\title{
Spin-Exchange Collisions of Submerged Shell Atoms Below 1 Kelvin
}

\section{Citation}

Harris, J. G. E., S. V. Nguyen, S. C. Doret, W. Ketterle, and J. M. Doyle. 2007. “Spin-Exchange Collisions of Submerged Shell Atoms Below 1 Kelvin." Physical Review Letters 99 (22) (November 30). doi:10.1103/physrevlett.99.223201.

\section{Published Version}

doi:10.1103/PhysRevLett.99.223201

\section{Permanent link}

http://nrs.harvard.edu/urn-3:HUL.InstRepos:27868186

\section{Terms of Use}

This article was downloaded from Harvard University's DASH repository, and is made available under the terms and conditions applicable to Other Posted Material, as set forth at http:// nrs.harvard.edu/urn-3:HUL.InstRepos:dash.current.terms-of-use\#LAA

\section{Share Your Story}

The Harvard community has made this article openly available.

Please share how this access benefits you. Submit a story.

\section{Accessibility}




\title{
Spin-Exchange Collisions of Submerged Shell Atoms Below 1 Kelvin
}

\author{
J. G. E. Harris, ${ }^{1}$ S. V. Nguyen, ${ }^{2}$ S. C. Doret, ${ }^{2}$ W. Ketterle,,${ }^{3,4}$ and J. M. Doyle ${ }^{2,4}$ \\ ${ }^{1}$ Departments of Physics and Applied Physics, Yale University, New Haven, Connecticut 06520, USA \\ ${ }^{2}$ Department of Physics, Harvard University, Cambridge, Massachusetts 02138, USA \\ ${ }^{3}$ Department of Physics, MIT, Cambridge, Massachusetts 02139, USA \\ ${ }^{4}$ Harvard / MIT Center for Ultracold Atoms, Cambridge, Massachusetts 02138, USA
}

(Received 4 May 2007; published 30 November 2007)

\begin{abstract}
Angular momentum changing collisions can be suppressed in atoms whose valence electrons are submerged beneath filled shells of higher principle quantum number. To determine whether spin-exchange collisions are suppressed in these "submerged shell" atoms, we measured collisional rates for six hyperfine states of $\mathrm{Mn}$ at $T<1 \mathrm{~K}$. Although the $3 d$ valence electrons in $\mathrm{Mn}$ are submerged beneath a filled $4 s$ orbital, we find spin-exchange rate coefficients similar to $\mathrm{Na}$ and $\mathrm{H}$ (both nonsubmerged shell atoms).
\end{abstract}

Quantum degenerate atomic gases are most commonly realized by evaporatively cooling atoms in a magnetic trap. Efficient evaporative cooling requires that angular momentum changing collisions (which can be driven by dipolar, spin-exchange (SE), second-order spin-orbit, and anisotropic electrostatic interactions [1-4]) occur at much lower rates than elastic collisions. This requirement is usually met by trapping a species with a small magnetic moment (to minimize dipolar interactions) and a ground state with orbital angular momentum $L=0$ (removing anisotropic electrostatic interactions to lowest order). These atoms are then trapped in "stretched" hyperfine states which are immune to SE relaxation. Alkali atoms meet these criteria and are by far the most widely-studied atoms in the ultracold regime.

Achieving quantum degeneracy in a wider range of species would enable the study of new types of quantum fluids [5], quantum computing architectures [6], and searches for time dependence of fundamental constants [7]. As a step towards this goal, recent work [8-10] showed that some angular momentum changing collisions are suppressed in "submerged shell" atoms - species whose valence electrons lie at smaller radii than filled shells of higher principal quantum number. In collisions between He and a submerged shell atom with $L \neq 0$, the filled outer shells shield the anisotropic electrostatic interaction, reducing the rate of angular momentum changing collisions by $10^{4}-10^{6}$. As a result, $L \neq 0$ submerged shell atoms could be cooled via a He buffer gas while maintaining the orientation of their magnetic moment. Atomic Tm, Er, $\mathrm{Nd}, \mathrm{Tb}, \mathrm{Pr}, \mathrm{Ho}$, and Dy ( $4 f$ valences shielded by filled $5 s$ and $6 s$ shells) were all magnetically trapped for the first time using this approach [10]. Collisions between He and Ti ( $3 d$ valence shielded by filled $4 s$ shell) showed a comparable suppression of the anisotropic electrostatic interaction $[8,9]$.

It is an open question whether a submerged valence suppresses other types of angular momentum changing collisions, such as SE. Another open question is whether a submerged valence suppresses angular momentum changing collisions of pairs of submerged shell atoms (as opposed to collisions between He and a submerged shell atom, as in Refs. [8-10]). These questions are central to determining whether submerged shell effects can extend the range of atomic species and hyperfine states which can be efficiently evaporatively cooled. They are also interesting from the point of view of atomic collisions generally.

To address these questions, we trapped several hyperfine states of ${ }^{55} \mathrm{Mn}$, which has a half-filled $3 d$ valence within a filled $4 s$ shell [11]. We determined SE and dipolar rate coefficients from trap losses. Unlike previous studies of collisions involving submerged shells [8-10], Mn has $L=$ 0 , leading to isotropic electrostatic interactions. This allows us to focus more cleanly on SE. Also, unlike previous studies, we measure collisions between submerged shell atoms. By working at relatively high temperature where many partial waves contribute to collisions, our results are more likely to reflect generic atomic structure effects than measurements in the ultracold regime which may be sensitive to "accidental" cancellations of a single partial wave [12].

Mn has a ${ }^{6} S_{5 / 2}$ ground state. The $S=5 / 2$ electronic spin couples to the $I=5 / 2$ nuclear spin via hyperfine. Figure 1(a) shows the calculated $B$ field dependence of the ground electronic manifold [13]. In our experiment, the majority of the atoms are in fields where $\varepsilon \equiv\left|a / g \mu_{B} B\right| \ll$ $1(a=-72.4 \mathrm{MHz}$ is the Mn hyperfine constant and $g=$ 2.00), so the electron and nuclear spin projections $m_{S}$ and $m_{I}$ are nearly good quantum numbers. We label the eigenstates $|1\rangle$ to $|36\rangle$ as shown in Fig. 1(a).

Mn was ablated, cooled, and trapped using a ${ }^{3} \mathrm{He}$ buffer gas apparatus described elsewhere [Fig. 1(b)] [12-16]. Once loaded into the anti-Helmholtz magnetic trap, the lifetime of trapped $\mathrm{Mn}$ in the most low-field seeking states (i.e., those with $m_{S}=5 / 2$ ) was not limited by collisions with background ${ }^{3} \mathrm{He}$. However, the ${ }^{3} \mathrm{He}$ density was kept 

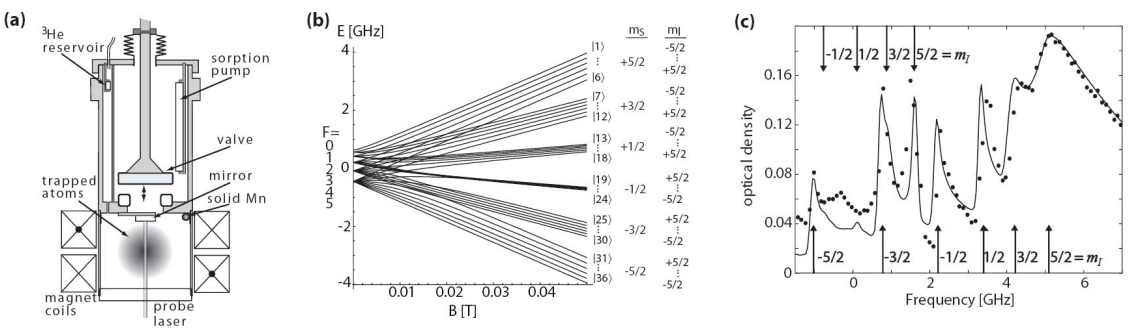

FIG. 1 (color online). (a) Schematic of the experimental cell. The cell is thermally linked to a ${ }^{3} \mathrm{He}$ refrigerator. For each measurement, the lower chamber is filled with ${ }^{3} \mathrm{He}$ from the reservoir and solid $\mathrm{Mn}$ is ablated. Then the valve is opened, and the ${ }^{3} \mathrm{He}$ is removed by the sorption pump. (b) The ground state manifold of ${ }^{55} \mathrm{Mn}$. The Zeeman splitting of the ground state is shown, along with the quantum numbers in the small- and large- $B$ limits. (c) Spectrum of trapped Mn. The solid line is a fit including the MaxwellBoltzmann distribution of the atoms in the trap, the radius of the probe beam, its offset from the trap center, the orientation of its polarization, the atoms' Doppler and lifetime broadening, and the optical transitions' Clebsch-Gordan coefficients. The location of each peak is labeled by the $m_{I}$ value of the ground state. Labels on the upper (lower) axis indicate $\Delta m=0(+1)$ transitions.

high enough so that the Mn remained in thermal contact with the cell walls. The populations of the trapped states were monitored by absorption spectroscopy on the ${ }^{6} S_{5 / 2}-$ ${ }^{6} P_{7 / 2}$ transition.

Figure 1(c) shows a typical spectrum of trapped Mn. The data are fit (solid line) by calculating the absorption for transitions between the ${ }^{6} S_{5 / 2}$ ground and ${ }^{6} P_{7 / 2}$ excited state manifolds [17]. The fit includes transitions only from states $|1\rangle-|6\rangle$, i.e., the maximally trapped $m_{S}=5 / 2$ states. No spectroscopic evidence of other states was seen.

Figure 2 shows the time dependence of the six trapped states populations. Each population is extracted from spectra and fits as in Fig. 1(c). The data in Fig. 2(a) were taken at $T=855 \mathrm{mK}$ and trap depth $B_{\text {trap }}=3.9 \mathrm{~T}$, while for Fig. 2(b), $T=480 \mathrm{mK}$ and $B_{\text {trap }}=2.0 \mathrm{~T}$, giving identical values of $\eta \equiv g m_{S} \mu_{B} B_{\text {trap }} / k_{B} T$. This ensures that atom loss over the top of the trap is the same in both data sets and that differences in atom loss reflect the $B$ - and $T$-dependence of the atoms' collisional properties. Fits to the spectra show the atoms remain in good thermal contact with the cell walls.

We begin by noting the qualitative aspects of Fig. 2 . First, the decay does not have the exponential form expected for loss due to background gas. This is consistent with the large mass ratio between ${ }^{55} \mathrm{Mn}$ and ${ }^{3} \mathrm{He}$ and the fact that $\eta \gg 1$. The decay is subexponential, consistent with loss via Mn-Mn collisions. The loss is state-dependent with the stretched state $|6\rangle$ (corresponding to $m_{I}=5 / 2$ ) decaying slowest and lower $m_{I}$ states tending to decay faster. Figure 2 shows this state dependence is stronger at lower $T$ and $B$. Lastly, we do not observe any states from the $m_{S}<5 / 2$ manifolds.

To understand these features, we note that inelastic MnMn collisions are expected to be mostly magnetic dipole and SE in origin. Since $\varepsilon \ll 1$ in most of the trap, we first consider the limit $\varepsilon=0$. In this limit, the atomic eigenstates are exact $m_{S}, m_{I}$ eigenstates. The rate coefficients for dipolar relaxation are the same for each state in the $m_{S}=$
$5 / 2$ manifold, and there is no SE relaxation. States in the $m_{S}=3 / 2$ manifold are not protected against SE, consistent with their absence from the trap. It is easy to show that if $\varepsilon=0$, the six populations remain in fixed ratio and
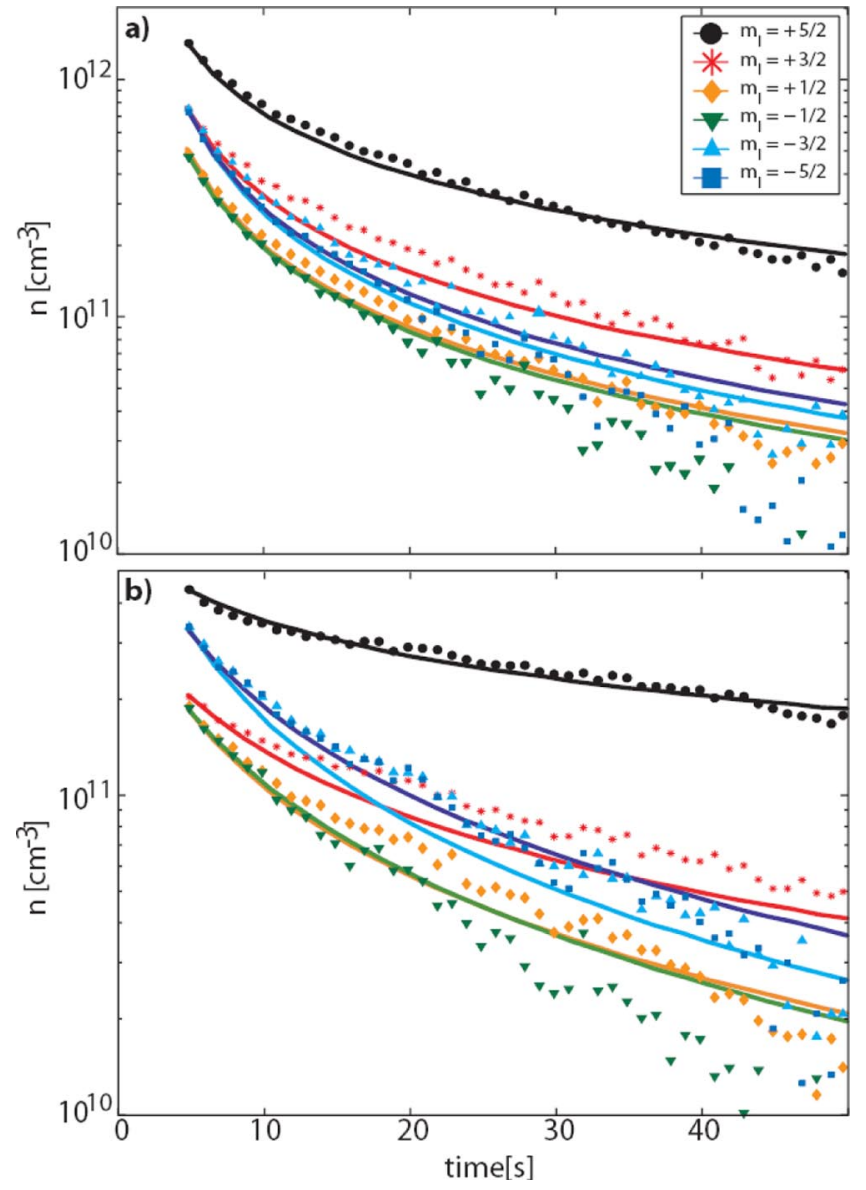

FIG. 2 (color online). Trap loss of the various hyperfine states. (a) Peak density $n(t)$ of each of the six hyperfine states in the trap versus time at $T=855 \mathrm{mK}$ and $B_{\text {trap }}=3.9 \mathrm{~T}$. The lines are fits described in the text. (b) same as (a) but for $T=480 \mathrm{mK}$ and $B_{\text {trap }}=2.0 \mathrm{~T}$. 
decay as $n_{z}(t)=n_{z}(0) /\left[1+\frac{1}{8} n_{\text {tot }}(0) \Gamma_{d} t\right]$, where $n_{z}(t)$ is the peak density of atoms in state $|z\rangle, n_{\text {tot }}=\sum_{z} n_{z}, z$ runs from 1 to 6 , and $\Gamma_{d}$ is the dipolar relaxation rate coefficient.

For the more realistic case $0<\varepsilon \ll 1$, the atomic eigenstates are not exact $m_{S}, m_{I}$ eigenstates (except for the stretched state): they acquire a component $\propto \varepsilon$ from the neighboring $m_{S}$ manifolds. Because the trapped states (and the states to which they relax) are all modified differently by this hyperfine-induced mixing, the dipolar relaxation is state-dependent. Additionally, this mixing leads to SE relaxation from the $m_{S}=5 / 2$ manifold. This SE relaxation is also state-dependent, and is zero for the stretched state.

Thus, we expect the rate coefficients to be dominated by the bare (i.e., hyperfine-unmodified, state-independent) dipolar relaxation $\left(\Gamma_{d}\right)$, and to have state-dependent corrections due to SE and hyperfine-modified dipolar. Both corrections $\propto \varepsilon^{2}$ (the relevant matrix elements $\propto \varepsilon$ ), and are smallest for the stretched state. This is qualitatively consistent with the data in Fig. 2; as $\varepsilon$ increases ([from Fig. 2(a) to Fig. 2(b)], the trap loss becomes more state-dependent, and the stretched state always has the lowest loss.

While this qualitative treatment explains several features of the data, it does not allows to us to distinguish hyperfinemodified dipolar from SE relaxation or to reach any conclusion about suppression of SE by Mn's submerged valence. To refine our analysis, we estimate the hyperfine corrections to dipolar relaxation (ignoring SE for the present). The matrix element for dipolar transitions from initial spin states $|x\rangle,|y\rangle$ to final spin states $\left|x^{\prime}\right\rangle,\left|y^{\prime}\right\rangle$ is

$$
V_{x, y, x^{\prime}, y^{\prime}}=\left\langle x^{\prime}, y^{\prime}\left|V_{d}\right| x, y\right\rangle
$$

where $V_{d}=\mathbf{S}_{1} \cdot \mathbf{S}_{2}-3\left(\mathbf{S}_{1} \cdot \hat{\mathbf{r}}\right)\left(\mathbf{S}_{2} \cdot \hat{\mathbf{r}}\right)$ is the spin component of the dipolar interaction and $\hat{\mathbf{r}}$ is the interatomic unit vector. $V_{d}$ implicitly includes the second-order spin-orbit interaction, which has the same spin dependence as dipolar. Ideally, one would include the initial and final states' wave vectors ( $k$ and $\left.k^{\prime}\right)$ in Eqn. (1) and integrate over the atoms' Boltzmann distribution, but as a first approximation, we ignore the collisions' $k$-dependence. We write $|x\rangle$, $|y\rangle,\left|x^{\prime}\right\rangle$, and $\left|y^{\prime}\right\rangle$ in the $m_{S}, m_{I}$ basis and calculate $V_{x, y, x^{\prime}, y^{\prime}}$ to first order in $\varepsilon$. We ignore symmetrization of the twoatom states because of the many partial waves and hyperfine states in the trap.

The rate coefficient for changes in the population of $|z\rangle$ due to collisions between atoms in $|x\rangle$ and $|y\rangle$ is $\Gamma_{x, y}^{(z)} \propto$ $\sum_{\left\{x^{\prime}, y^{\prime}\right\}}\left|V_{x, y, x^{\prime}, y^{\prime}}\right|^{2} \Lambda$ where the constant of proportionality is the same for all $\Gamma_{x, y}^{(z)}$ and is absorbed into the fitting parameters described below. $\Lambda \equiv \delta_{x^{\prime}, z}+\delta_{y^{\prime}, z}-\delta_{x, z}-\delta_{y, z}$ counts the change in the population of $|z\rangle$ for each term in the sum. The sum is over the distinguishable pairs of $x^{\prime}$ and $y^{\prime}$ which each run from 1 to 36 to account for all possible inelastic processes.
For two-body loss processes, the six rate equations for $z=1-6$ can be written in terms of the $\Gamma_{x, y}^{(z)}$ :

$$
\dot{n}_{z}=\sum_{\{x, y\}} \Gamma_{x, y}^{(z)} n_{x} n_{y} .
$$

We solve Eqn. (2) numerically (using the earliest measured $n_{z}(t)$ as the initial condition) to fit the data in Fig. 2.

As discussed above, if $\varepsilon=0$, the atomic eigenstates are $m_{S}, m_{I}$ eigenstates and $\Gamma_{x, y}^{(z)}=\Gamma_{d}$ for all $x, y, z$. Evaluating Eqn. (1) to first order in $\varepsilon$ gives the fractional, statedependent, hyperfine-induced, correction to $\Gamma_{d}$, but not $\Gamma_{d}$ itself. Thus, we can write all the rate coefficients in Eqn. (2) in terms of a single unknown, $\Gamma_{d}$, which we use as a fitting parameter.

Because $\varepsilon$ varies throughout the trap, the $\Gamma_{x, y}^{(z)}$ are position dependent. Averaging the $\Gamma_{x, y}^{(z)}$ over the atoms' Boltzmann distribution gives the usual factor of $\frac{1}{8}$ for the $B$-independent terms (the hyperfine-unmodified dipolar contribution), while the $B$-dependent $\left(\propto \varepsilon^{2}\right)$ terms lead to an "average" $\varepsilon_{\text {avg }}=a m_{S} / 2 k_{B} T$.

The qualitative trends in the trap loss are not reproduced by fitting the data to Eqn. (2) using the hyperfine-modified dipolar relaxation rate coefficients. Most importantly, this model leads to trap loss in which states with lower $\left|m_{I}\right|$ decay fastest, while in Fig. 2 states with lower $m_{I}$ decay fastest. This disagreement suggests that relaxation processes other than dipolar (such as SE) are present.

To treat SE at the same level of approximation, we repeat the calculation of $\Gamma_{x, y}^{(z)}$ but replace $V_{d}$ in Eqn. (1) with $a V_{d}+b V_{s}$, where $a$ and $b$ are scalar constants and $V_{s}=\mathbf{S}_{1} \cdot \mathbf{S}_{2}$ is the spin component of the SE interaction. This gives new rate coefficients for Eqn. (2) in terms of two unknowns: $\Gamma_{d}$ (characterizing the strength of the dipolar relaxation) and $\Gamma_{s}$ (which characterizes the strength of the SE relaxation, and so should be sensitive to suppression of SE).

Using both $\Gamma_{d}$ and $\Gamma_{s}$ as fit parameters greatly improves the agreement with the data, capturing the important features in Fig. 2 such as states with lower $m_{I}$ decaying faster and the fact that this behavior is more pronounced at lower $T$ (i.e., higher $\varepsilon_{\text {avg }}$ and more hyperfine-induced mixing).

This model is valid only for $\varepsilon \ll 1$. This condition holds for the vast majority of the atoms, but the atom density and hyperfine-induced mixing are both highest at the trap center. Thus, for a small fraction of the atoms, $\varepsilon$ is no longer $\ll 1$, and the spin wave functions used above are not accurate. Extending our perturbation theory approach to higher orders would lead to new relaxation channels and so increased state-dependent trap loss. As a result, the effective spatially-averaged value of $\varepsilon$ should be somewhat greater than $\varepsilon_{\text {avg }}$. To account for this, we also fit the data using $\varepsilon$ as a fitting parameter. The result is shown as the solid lines in Fig. 2. The fit in Fig. 2(a) gives $\Gamma_{d}=3.0 \times$ $10^{-13} \mathrm{~cm}^{3} / \mathrm{s}, \quad \Gamma_{s}=2.2 \times 10^{-14} \mathrm{~cm}^{3} / \mathrm{s}$, and $\varepsilon=0.009$ 
(the $a b$ initio value $\varepsilon_{\text {eff }}=0.0047$ ). The fit in Fig. 2(b) gives $\Gamma_{d}=1.1 \times 10^{-13} \mathrm{~cm}^{3} / \mathrm{s}, \quad \Gamma_{s}=4.0 \times 10^{-14} \mathrm{~cm}^{3} / \mathrm{s}$, and $\varepsilon=0.028\left(\varepsilon_{\text {eff }}=0.0085\right)$. The values of $\Gamma_{d}$ are slightly smaller than for ${ }^{52} \mathrm{Cr}$ under similar circumstances [12], consistent with the relative magnetic moments of $\mathrm{Mn}$ and $\mathrm{Cr}$. $\Gamma_{s}$ is the typical contribution to $\Gamma_{x, y}^{(z)}$ due only to SE. The actual SE contribution to each $\Gamma_{x, y}^{(z)}$ is $d_{x, y, z} \Gamma_{s}$ where $-1 \leq$ $d_{x, y, z} \leq 2$ is determined from the perturbation theory calculation described above.

Using $\varepsilon$ as a fitting parameter is an ad hoc solution to the breakdown of perturbation theory at the trap center. However, the fact that it gives an effective $\varepsilon$ somewhat greater than $\varepsilon_{\text {eff }}$ is at least consistent with our understanding of this breakdown. A more complete treatment would need to consider the full Zeeman structure of the ground state manifold, a task beyond the scope of this Letter.

The fits in Fig. 2 extrapolate to unequal populations at $t=0$, contrary to what one would expect for thermal equilibrium. However, in the seconds after ablation (at $t=$ 0 ), the system may be far from equilibrium - the cell cools and the ${ }^{3} \mathrm{He}$ density drops many orders of magnitude. Thus, extrapolations of the fits are not likely to be valid during this period. Data and fits are shown for $t>4 \mathrm{~s}$, when we believe the system is close to equilibrium.

To see whether the SE rate coefficients inferred from Fig. 2 are influenced by the submerged valence of $\mathrm{Mn}$, we must compare them with nonsubmerged-shell atoms at similar values of $\varepsilon$. This is possible for $\mathrm{Na}$, where the rate coefficient $\Gamma_{6,7, s}^{(7), \mathrm{Na}}$ for SE collisions between $\mathrm{Na}$ atoms in states $|6\rangle_{\mathrm{Na}}$ and $|7\rangle_{\mathrm{Na}}$ (which in the $\left|m_{S}, m_{I}\right\rangle$ basis asymptote to $\left|\frac{1}{2}, \frac{1}{2}\right\rangle$ and $\left.\left|\frac{1}{2},-\frac{1}{2}\right\rangle\right)$ have been calculated [18]. Extrapolating the results of Ref. [18] gives $\Gamma_{6,7, s}^{(7), N a}=$ $4 \times 10^{-15} \mathrm{~cm}^{3} / \mathrm{s}$ for $\varepsilon=0.009$, equivalent to Fig. 2(a), and $\Gamma_{6,7, s}^{(7), \mathrm{Na}} \approx 4 \times 10^{-14} \mathrm{~cm}^{3} / \mathrm{s}$ for $\varepsilon=0.028$, equivalent to Fig. 2(b).

We take the Mn states $|5\rangle$ and $|4\rangle$ as the equivalent states - i.e., those with one and two lower $m_{I}$ than the stretched state [Fig. 1(a)]. The component of the rate coefficient $\Gamma_{5,4}^{(5)}$ due only to $\mathrm{SE}$ is $\Gamma_{5,4, s}^{(5)}=d_{5,4,5} \Gamma_{s}=1.2 \times$ $10^{-14} \mathrm{~cm}^{3} / \mathrm{s}$ (for $\varepsilon=0.009$ ) and $2.2 \times 10^{-14} \mathrm{~cm}^{3} / \mathrm{s}$ (for $\varepsilon=0.028)$. Thus, the rate coefficients for Mn are comparable to $\mathrm{Na}$ (though the $\mathrm{Na}$ values are for $T=0$ and only a single partial wave). Calculations of SE in atomic $\mathrm{H}$ also give rate coefficients comparable to those measured here for $\mathrm{Mn}$ [19].

This suggests that the submerged valence of Mn does not strongly suppress the SE contribution to trap loss. However, SE leads to trap loss via the phase difference between collisions on different molecular potentials (for $\mathrm{Mn}$, the two relevant potentials are those with total spin $S_{M}=5 \& 4$ ). It is conceivable that the submerged valence does suppress SE, but not enough to give a phase shift $\ll 2 \pi$ between the $S_{M}=5 \& 4$ potentials. Accurate cal- culations of the Mn-Mn potentials would allow for more quantitative conclusions.

Whether the results of this work are general or merely peculiar to Mn could be tested by repeating the same measurements and analysis for other $L=0, I \neq 0$ atoms and comparing the results for submerged valences (Re and Eu) with nonsubmerged (alkalis, coinage metals, ${ }^{53} \mathrm{Cr}$, ${ }^{95} \mathrm{Mo}$, and ${ }^{97} \mathrm{Mo}$ ). It may be that rare-earth atoms (whose $4 f$ valences are shielded by both $5 s$ and $6 s$ orbitals) achieve stronger SE suppression than Mn. Again, detailed calculations of these species' interatomic potentials could shed considerable light on this question.

A source of trap decay not included in our analysis is Majorana loss. The usual Majorana loss at the trap center should be negligible, but Fig. 1(a) shows that states $|3\rangle-|5\rangle$ cross $m_{S}=3 / 2$ states at finite $B$ and so should undergo Majorana transitions at spherical surfaces in addition to the trap center ( $|6\rangle$ does not mix with $m_{S}=3 / 2$ states and so is immune to this process). Interestingly, $|1\rangle$ has no level crossings, even at $B=0$, and so does not have any Majorana losses, even in an anti-Helmholtz trap. While the trap loss due to these crossings is difficult to estimate $a$ priori, we see no sign of enhanced loss for states $|3\rangle-|5\rangle$. Rather, states $|1\rangle$ and $|2\rangle$ decay fastest.

We acknowledge discussions with Roman Krems, Jonathan Weinstein, David Demille, and Robert Michniak. This work was done at Harvard University as part of the NSF Center for Ultracold Atoms.

[1] A. Lagendijk, I. F. Silvera, and B. J. Verhaar, Phys. Rev. B 33, 626 (1986).

[2] W. Ketterle and N. J. VanDruten, Adv. At. Mol. Opt. Phys. 37, 181 (1996).

[3] R. V. Krems, G.C. Groenenboom, and A. Dalgarno, J. Phys. Chem. A 108, 8941 (2004).

[4] P. J. Leo et al., Phys. Rev. Lett. 81, 1389 (1998).

[5] L. Santos et al., Phys. Rev. Lett. 85, 1791 (2000).

[6] A. Derivianko and C. C. Cannon, Phys. Rev. A 70, 062319 (2004).

[7] A. T. Nguyen et al., Phys. Rev. A 69, 022105 (2004); V. A. Dzuba et al., Phys. Rev. A 68, 022506 (2003).

[8] C. I. Hancox et al., Phys. Rev. Lett. 94, 013201 (2005).

[9] R. V. Krems et al., Phys. Rev. Lett. 94, 013202 (2005).

[10] C. I. Hancox et al., Nature (London) 431, 281 (2004).

[11] S. R. Langhoff and C. W. Bauschlicher, Jr., Annu. Rev. Phys. Chem. 39, 181 (1988).

[12] P. S. Julienne et al., Phys. Rev. Lett. 78, 1880 (1997).

[13] S. V. Nguyen, Ph.D. thesis, Harvard University, 2006.

[14] J. G. E. Harris et al., Rev. Sci. Instrum. 75, 17 (2004).

[15] J. G. E. Harris et al., Europhys. Lett. 67, 198 (2004).

[16] S. V. Nguyen et al., Phys. Rev. A 71, 025602 (2005).

[17] J. D. Weinstein, Ph.D. thesis, Harvard University, 2001.

[18] E. Tiesinga et al., Phys. Rev. A 43, 5188 (1991).

[19] H. T. C. Stoof, J. M. V. A. Koelman and B. J. Verhaar, Phys. Rev. B 38, 4688 (1988). 